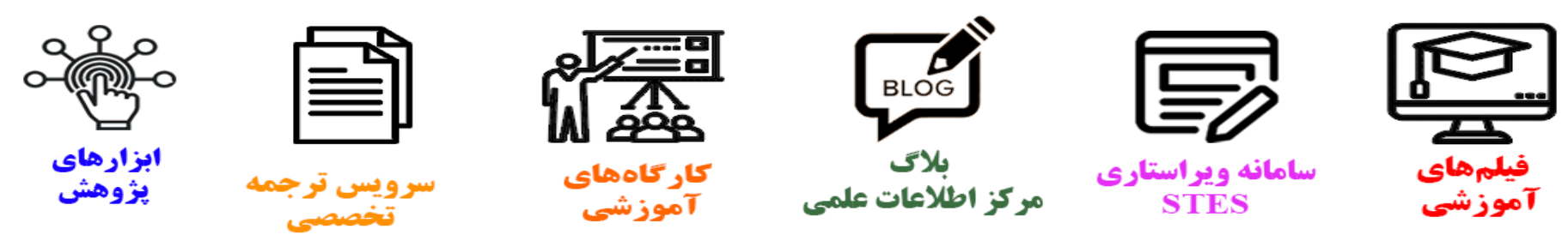

\title{
(c)
}

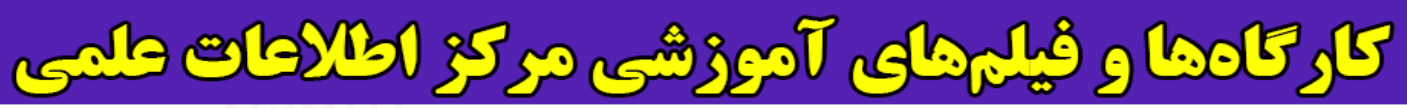
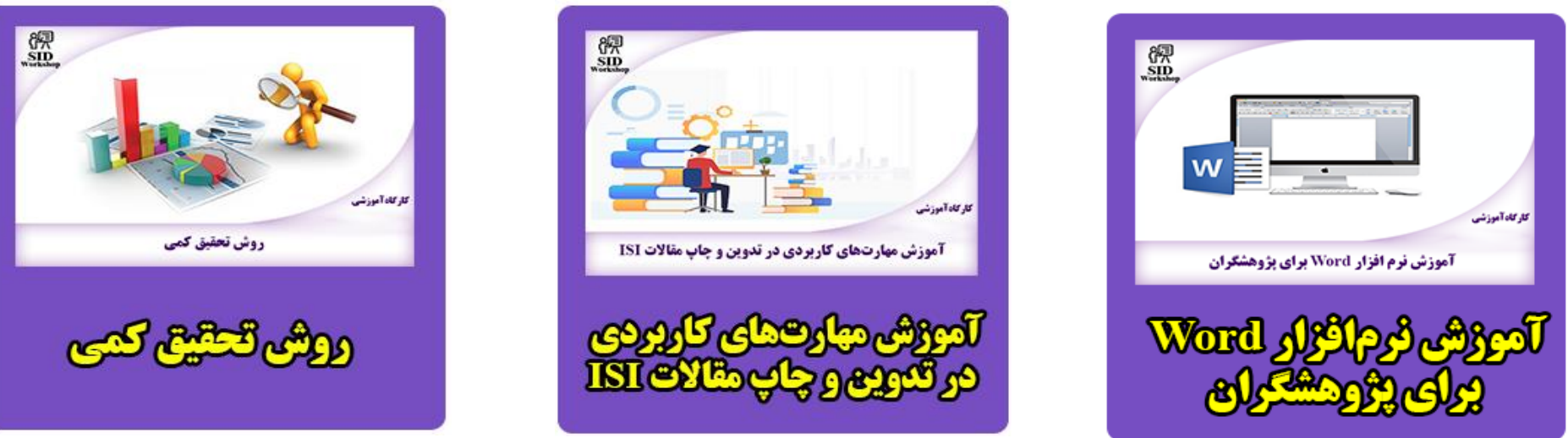


\section{Giant Endobronchial Inflammatory Polyp}

M. Sokouti, ${ }^{1}$ F. Ghavam, ${ }^{2}$ V. Montazeri ${ }^{1}$

${ }^{1}$ Department of Thoracic Surgery,

Tabriz University of Medical Sciences, Tabriz, Iran.

${ }^{2}$ Department of Pathology,

Urmia University of Medical Sciences,

Urmia, Iran.

\section{Correspondence:}

Mohsen Sokouti MD,

Department of Thoracic Surgery,

Imam Khomeini Hospital,

Tabriz University of Medical Sciences,

Tabriz, Iran.

Tel/Fax: +98 4113347054

E mail: sokouti_m@yahoo.com

\begin{abstract}
Inflammatory endobronchial polyp is a rare disease mostly encountered in asthmatic patients. Chronic airway and foreign material irritation or thermal injury may result in the formation of granulated tissues and become polypoid mass. Herein, we describe a 52-year-old man with severe respiratory distress and infection with 35 years history of smoking. He had an obstructive pattern in his pulmonary function tests and severe bronchiectasis of right lower lobe that responded well to lobectomy with polypectomy. Pathologic examination revealed large endobronchial polyp 9x2 cm obstructing right and left bronchi and right lower lobe bronchiectasis. Such large inflammatory endobronchial polyp has rarely been presented in literature.
\end{abstract}

Iran J Med Sci 2005; 30(1): 45-47.

Keywords • Bronchus • Endobronchial • inflammatory polyp

\section{Introduction}

I nflammatory polyps (IP) of the airways are now regarded as histopathological distinct non neoplastic endobronchial lesions, which in adults are associated with a variety of chronic inflammatory insults. ${ }^{1}$ The benign endobronchial polyp was first described in 1930 and called "neoplasm" by Patterson. ${ }^{2}$ IP of the tracheobronchial tree are uncommon comprising only 7 out of $63(11 \%)$ benign tracheobronchial tumors in one series. Several pathologic mechanisms have been proposed. In some cases, a history of chronic bronchitis, asthma or bronchiectasis has suggested that the polyps represent an exaggerated but localized inflammatory reaction to chronic airway irritation. ${ }^{1,3,4}$ In others, aspirated foreign materials or thermal injury has resulted in the form of exuberant granulation tissue that constitutes the polypoid mass. They can be treated by endoscopic ablation, simple or laser, or by surgery if the lung is destroyed. ${ }^{2,5}$ Herein, we report a large $(9 \times 2$ $\mathrm{cm}$ ) inflammatory bronchial polyp that produced severe dyspnea and right lower lobe bronchiectasis and treated by surgery. Up to now, only one such a large endobronchial polyp is reported in the literature. ${ }^{6}$

\section{Case Presentation}

A 52-year-old man was admitted to Motaheri hospital affiliated to Urmia University of Medical Sciences, Urmia, Iran, with two years history of right lower lobe pneumonia. The patient had malaise, severe dyspnea, tachypnea and restlessness. He was receiving oral and intravenous antibiotics irregularly for two years for pneumonia. His conditions were worsening with shallow breathing, severe dyspnea, right lower chest pain, and fever associated with daily expectoration of large yellow sputum, acrocyanosis, fatigue and streaky hemoptysis. He was well 


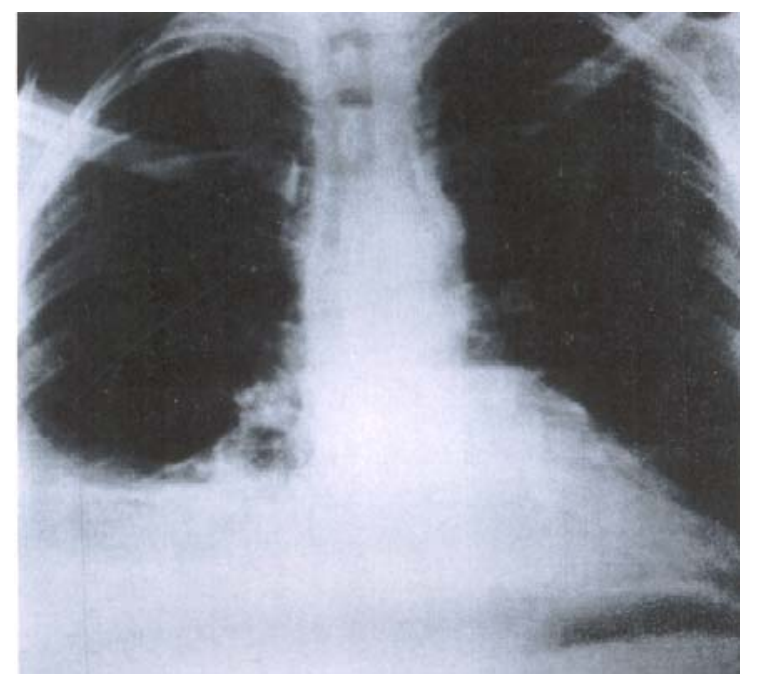

Fig1: Chest X-Ray revealed honey comb appearance and right lower lobe consolidation.

nourished but had a history of 70 pack year smoking. On examination besides coughing, he had severe respiratory distress and dyspnea using his intercostals and supraclavicular muscles on respiration. He had a respiratory rate of $35 / \mathrm{min}$, heart rate 79 beats $/ \mathrm{min}$, and his blood pressure was $120 / 70 \mathrm{mmHg}$ with the body temperature of $38^{\circ} \mathrm{C}$.

There were no abnormal findings on examinations of head, neck, extremity, heart, and his ECG did not show any changes in cardiopulmonary system. On auscultation, he had decreased respiratory sounds, dullness with fine and coarse crackles in right lower hemithorax, and wheezing in both lungs. Cytology of the sputum was normal. His chest X-ray revealed atelectasis of the right middle lobe and a densely honey comb appearance mass observed in the right lower lobe and costophrenic triangle suggesting a pulmonary sequestration with fibrotic changes and bronchiectasis (Fig 1).

On admission the impression was that, the patient had severe bronchiectasis of the right lower lobe with hemoptysis, shortness of breath and severe obstructive lung disease. The patient underwent fiber optic flexible bronchoscopy which revealed a smooth and reddish mass on carina that occluded $70 \%$ of right and $40 \%$ left bronchi respectively. Biopsy was not performed in fear of bleeding because of high vascularization of mucosal surface of the mass. Since diagnosis was not achieved with flexible bronchoscopy the patient was referred to thoracic surgery ward. A cupped-forceps biopsy with rigid bronchoscopy was performed but no results was obtained, because the mass was firm, dodged and sprang away from the forceps with resilience or elasticity. Therefore, the patient received combination drug therapy with ceftazidim and clindamycin. After im-

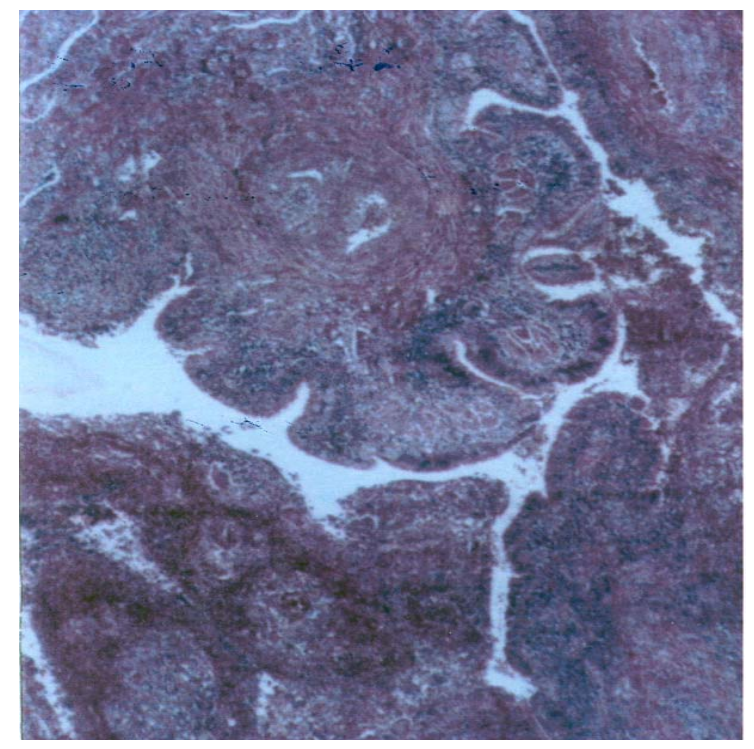

Fig 2: Microscopic appearance of endobronchial polyp, showing a polypoid mass lined with respiratory type epithelium consisting of granulating tissue and inflammatory process (x40).

provement and reduction of the sputum, bronchiectasis and pneumonia was diagnosed on exploration of right posterolateral thoracotomy.

A 3 to $4 \mathrm{~cm}$ right bronchotomy revealed 70 to $80 \%$ occlusion of the right main stem of bronchus accompanied with atelectasis of the right lower lobe. A large polyp mass measuring $9 \times 2 \mathrm{~cm}$ that protruded to carina and the left bronchus was delivered from the incision of the right bronchus. After opening left bronchus, one lung anesthesia was started and right lower lobe resected with a giant polyp on it. Bronchiectasis was present in the involved areas of the right lower lobe and pathologic examination revealed inflammatory endobronchial polyp (Fig 2). There was not any operative or postoperative complication. On day-15 of operation the patient was discharged from the hospital with good functioning respiratory system, and one-year follow up did not show any post-operative complication.

\section{Discussion}

Benign polyps of the endobronchial tree are notably rare. They are smooth, pink, conical shaped, and have a relatively narrow base covered with normal looking- mucosa. ${ }^{1}$ They have no erythema, ulceration, nodularity, necrosis, and are associated with visible vascular abnormality. Their appearance does not seem inflammatory and they are not the source of bleeding. Biopsy could not be performed on them because of elasticity of polyp that experimentally associated with more healthy tissue. The histology of the polyp revealed squamous 
metaplasia which is the most frequent pathologic description of benign polyps that encountered in the literature. In our case a solitary large polyp measuring $9 \times 2 \mathrm{~cm}$ was located in the right main stem bronchus near a chronic locus of infection and bronchiectasis of right lower lobe. Our patient's age was 52 years and history of 70 pack year smoking with possible severe obstructive impairment of his lung and this made us reluctant to decide surgical approach to his problem. Management of single or multiple benign appearing polyps is unclear from the literature and possibly even controversial. ${ }^{1,2}$ Eppings stated outright, in 1988 that "surgical resection" is the chosen treatment mostly based on the rational that benign polyps are often indistinguishable from malignant tumor. ${ }^{2}$ More recently, some clinicians have found that these polyps may regress and disappear with medical treatment (inhaled steroids) and others may remove it endoscopically or by Laser therapy. ${ }^{3,7}$ There is nothing in the literature describing a benign tumor transforming into a malignant lesion. There is very little published on the natural course of these lesions or on whether surgical excision might be truly beneficial, although in severe bronchiectasis and destroyed lung it must be done. ${ }^{8}$

Sometimes the vasculature appearance of the tumor stimulates the bronchoscopist not to biopsy the lesion because of concern that the bleeding might be excessive and on occasions the tissue taken with the small forceps used with the flexible bronchoscopes does not provide as perceives that pathologist can properly characterize as a malignancy and deeper and larger biopsies are required. Rare other malignant tumors such as chondrosarcoma or paraganglionoma might be confused with benign polyp. Squamous epithelial papilloma (thought to be carcinoma) confused with benign lesion. Tracheopathica Osteoplastica has a polypoid appearance but it never involves membranous portion of the airway, only the cartilages. Granular cell myoblastoma of airway or endobronchial Hamartoma (Lipoma) are another differential diagnosis. $^{2}$

Here we report a large endobronchial polyp with severe respiratory symptoms that treated with surgery; a case that only reported once in the literature. ${ }^{6}$

\section{References}

1 Fraser, Muller, Colman, Pare: Diagnosis of diseases of the chest. Fourth ed. W,B. Saunders; 1999. p. 1371.

2 Schnader J, Harrell J, Mathur $\mathrm{P}$, et al. Clinical conference on management dilemmas: Bronchiectasis and endobronchial Polyps. Chest 2002; 121: 637-43.

3 Smith RE. Endobronchial Polyp and chronic injury. Postgrad Med J 1989; 65: 785-7.

4 Niimi A, Amitani R, Ikeda T, et al. inflammatory bronchial Polyps associated with asthma: resolution with inhaled corticosteroids. Eur Respir J 1995; 8: 1237-9.

5 Moisan TC. Retained endobronchial foreign body removal facilitated by steroid Therapy of an obstructing, inflammatory polyp. Chest 1991; 100: 270.

6 Vontz FK, Vitsky BH. Giant bronchial polyp treated by emergency thoracotomy. Chest 1974; 66: 102-4.

7 Snow N, Fratianne RB. Obstructing endobronchial inflammatory polyps: treatment with urgent Laser photoablation. J trauma 2001; 50: 753-4.

8 Levashev luN, Dvorakovskaia IV, Gerasin VA. Diagnosis and surgical treatment of benign endobronchial tumors of the trachea and bronchi. Vestn Khir Im I I Grek 1984; 133: 14-9. 


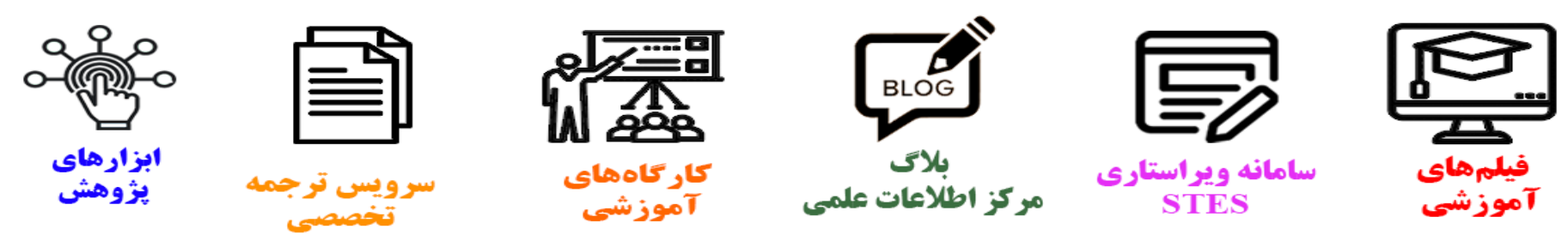

\section{(c)}

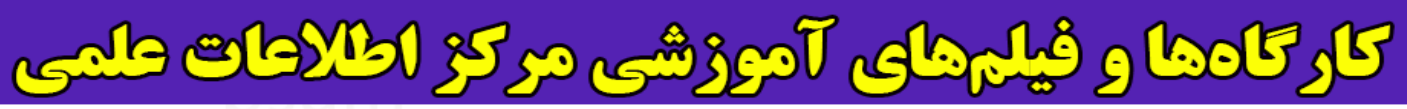
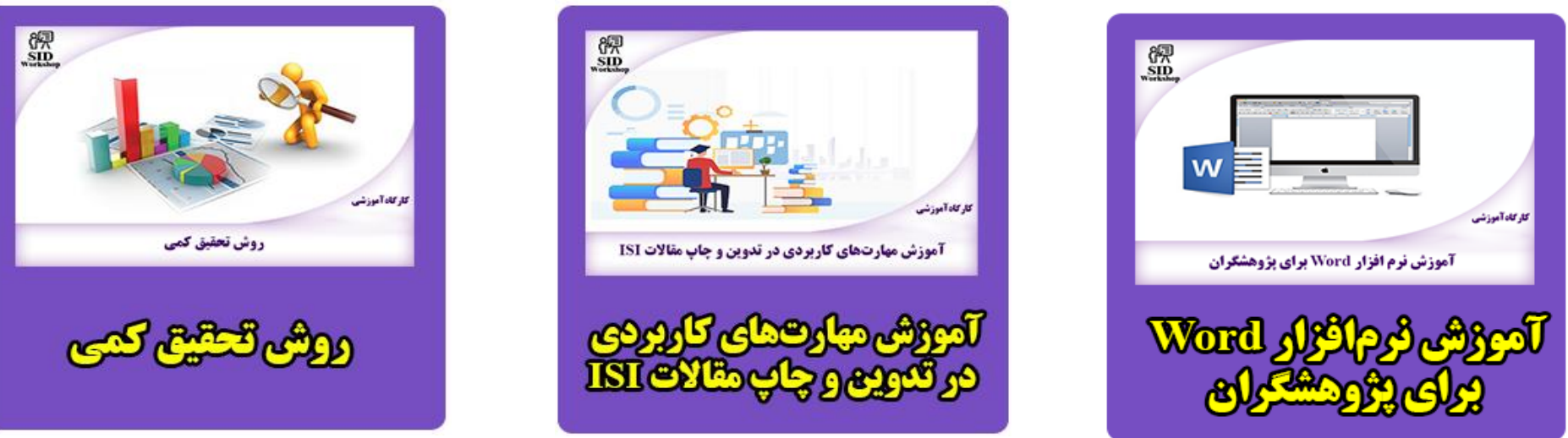